\title{
The role of sleep in processing emotional and contextual information: from mechanism to impact on everyday life and emotional health
}

\author{
María Corsi-Cabrera $\cdot$ Gina R. Poe
}

Published online: 23 April 2014

(C) Springer-Verlag Berlin Heidelberg 2014

In the last decade, a renewed interest has emerged regarding the association between sleep and emotional modulation/regulation. The aim of this special issue was to bring together, in a single issue, the current work of many scientists around the world. These laboratories are concerned with understanding the relationship between sleep and the processing of emotional information and with memory formation.

Converging evidence from human and animal studies has accumulated regarding the important role of sleep in general, and rapid eye movement (REM) sleep in particular, in the consolidation of emotional memories. Most of these studies have focused on the role of sleep in the processing of negative emotions as well as consolidating acquired information and regulating emotion the next day.

Different experimental approaches, including studies in humans and rats, have been used to reveal complex interactions between an endless cycle of waking pre-sleep experiences, sleep, and next day waking behavior. The disentanglement of the processes at work and their interactions is crucial for understanding the mechanisms whereby sleep affects emotional regulation. Development of effective treatments for pathophysiological conditions such as schizophrenia and mood disorders, especially post-traumatic stress disorder (PTSD), likely depends upon understanding

M. Corsi-Cabrera $(\bowtie)$

Facultad de Psicología, Universidad Nacional Autónoma de México, Posgrado, Laboratorio de Sueño, Av. Universidad 3004, 04510 Mexico, DF, Mexico

e-mail: corsi@unam.mx

G. R. Poe $(\bowtie)$

University of Michigan, Ann Arbor, MI, USA

e-mail: ginapoe@umich.edu the relationship between sleep, emotion, and memory processing.

Three review articles in this issue describe different sets of converging evidence that support the idea that REM sleep and the transition to REM sleep (non-REM sleep Stage 2) are essential to processing emotional loads. Even more specifically, traits within these states, such as sleep spindles, theta and gamma network oscillations, and the cessation of locus coeruleus noradrenergic firing are intimately linked to the function of sleep for emotional memory processing. These three theoretical constructs set the stage for understanding where each of the data articles in this issue fit into the bigger scheme.

The review by Deliens et al. (10.1007/s00221-0143832-1) provides an excellent panoramic view of current knowledge regarding the bidirectional interaction between sleep and emotional regulation. They provide a historical perspective of the growing comprehension in the field regarding how emotions modulate sleep architecture and dreaming, and how sleep or lack of it modulates next day brain activity and emotional reactivity. They stress the role of sleep in the recombination of memory elements, which allows a down-regulation of negative and arousing emotions that are linked to memories. That review is followed by a set of papers describing the effects of sleep deprivation on emotional and contextual processing. Next, a set of papers are presented that focus on traits of sleep that correlate with the best emotional outcome as well as with general memory consolidation processes that could influence emotional outcomes. These papers describe roles for slow wave sleep, spindle activity, REM sleep, theta, and gamma activity conservation in the brain. A review by Garcia-Rill (10.1007/s00221-013-3794-8) then ties together evidence in the literature regarding gamma generation, regulation, and implications for cognition and emotion. The last set 
of articles brings to light new evidence that sleep quality affects subsequent learning and emotional processing, and vice versa. A final review by Vanderehyden et al. (10.1007/ s00221-014-3890-4) links physiological sleep features common to PTSD in humans with animal studies, calling for more mechanistic detail and proposing a model that links locus coeruleus dysfunction with general memory consolidation issues, PTSD, and sleep disorders.

Data-based articles in the first set show that sleep functions to process previous experiences. Off-line processing of previous information during sleep facilitates consolidation of novel information, enhancing memory, and decreasing emotional reactivity. Chambers and Payne (10.1007/ s00221-013-3779-7) show how sleep enhances memory while decreasing emotional reactivity and adds a new twist by showing this relationship even for positive emotional (humorous) experiences. Through the sleep deprivation study of Cote et al. (10.1007/s00221-013-3780-1), we learn that sleep deprivation leads to deficits in categorizing and processing emotional expression, especially subtle and sad expressions in faces, while at the same time, sleep-deprived people show greater neural reactivity for threat-related negative emotions as perceived in fearful and angry faces.

Together the studies of Edward Pace-Schott, Amy Hunter, and Margarita Borquez support that idea that REM sleep improves the contextualization of memory, that is, the assignment of specific features and exceptions to cues that were once associated with psychological or physiological harm. Pace-Schott et al. (10.1007/s00221-014-38299) show that people who sleep after exposure to startling stimuli and after fear extinction training have lower skin responses and do not increase heart rate as much as those who do not sleep between exposure and habituation testing or between extinction training and testing. Thus, a good night's sleep following exposure to both startling stimuli and extinction training would enhance the therapeutic potential of these treatments. Hunter (10.1007/s00221-0143828-x) reports that REM sleep deprivation, in particular, impairs the consolidation of fear extinction training. Hunter was testing the hypothesis that co-housing versus isolated housing conditions would reduce the negative effects of sleep deprivation on extinction consolidation. Interestingly, although she did not find an overall housing effect, she did see that a naïve rat co-housed with a rat going through fear conditioning and extinction training more quickly learned and better remembered extinction of fear when they went through the protocol weeks later, while individually housed. Borquez et al. (10.1007/s00221-013-3797-5) demonstrate that sleep also enhances memory for non-emotional tasks demanding inhibitory behavioral control. An opportunity of as little as $80 \mathrm{~min}$ undisturbed sleep between training and testing gave rats better ability to discriminate a rewarded context from a new one. Their study results extend the known role for sleep in fear and extinction learning to other tasks involving prefrontal-hippocampal function. All three of the above-mentioned sleep versus no sleep studies indicate that sleep enhances habituation and extinction training, and allows better discrimination of context and reward or lack of punishment.

The findings in these first five data papers are consistent with the idea that sleep deprivation impairs the ability of people to distinguish fine subtleties in the context in which events occur and increases the impact of threats on the emotional system. Both impairments counter-indicate sleep disruption prior to threat exposure (e.g. the night before a planned battle) as it could increase susceptibility to PTSD.

The second section focuses on mechanisms. In it, several papers show that REM sleep or some trait of REM sleep mediates the extinction of fear memory. Fear extinction relies upon recognition of environmental differences, higher cognitive processing, and the knowledge of the context in order to decide whether to permit autonomic fear reactions such as racing heart and sweaty palms, or to prevent them. REM sleep seems to mediate this contextualization via the prefrontal cortex. Cowdin, Kobayashi, and Mellman (10.1007/s00221-014-3857-5) show that REM sleep-related theta activity in the right prefrontal cortex is higher in resilient individuals than in those that developed PTSD after a traumatic experience. The long lag between trauma and sleep measures that correlate with PTSD occurrence indicate that these electrophysiological features may represent long-term traits or perhaps even predictive biomarkers. McDevitt et al. (10.1007/s00221-014-3830-3) show that super learners of a complex perceptual learning task benefit greatly from REM sleep obtained in naps while normal learners benefit both from REM sleep and, surprisingly, active waking. They speculate that mechanisms common to waking and REM sleep may benefit consolidation. Mechanisms shared between the two states may include increased metabolic and electroencephalographic activity, and neuromodulatory features associated with synaptic plasticity such as cholinergic activity. The editors note that acetylcholine is involved in theta activity in memory association areas, thus mechanistically linking the Cowdin and McDevitt findings.

Another of several electrophysiologic and neurochemical features shared by both waking and REM sleep is gamma activity $(30-80 \mathrm{~Hz})$. Gamma activity is a signature of an activated brain state. The presence and synchrony of gamma activity have been shown to be involved in sensory perception, problem solving, and memory recall during waking. Gamma may serve similar functions during REM sleep. Interhemispheric synchrony or temporal coupling of EEG activity between the left and right hemispheres is enhanced during REM sleep compared to nonREM sleep and waking. Corsi-Cabrera et al. (10.1007/ 
s00221-013-3802-z) report a tight homeostatic regulation of gamma band temporal coupling between cortical hemispheres during REM sleep in humans. They speculate that the gamma coupling rebound consequence of REM sleep deprivation may be implicated in reestablishing the balance between left and right frontal regions involved in mood regulation and emotion by providing a brain state during REM sleep that favors interhemispheric communication. The review by Garcia-Rill et al. (10.1007/s00221013-3794-8) shows that although gamma may serve similar functions in both waking and REM sleep states, there are differences in intracellular pathways between gamma band activity during waking and REM sleep that may be involved in establishing the difference between dreaming and waking thinking. He discusses how gamma dysregulation between states may be involved in altered mental states such as schizophrenia.

Within non-REM sleep, Ward, Peters, and Smith (10.1007/s00221-013-3781-0) show that the density of sleep spindles is positively correlated with emotional declarative memory consolidation. During REM transition to REM sleep, containing a higher density of sleep spindles, during REM sleep, strong glutamatergic P-waves ascend from the locus subcoeruleus. Siwek et al. (10.1007/s00221-0143858-4) demonstrate P-waves are essential to the consolidation of context-based fear conditioning as their destruction, through small lesions of the nucleus subcoeruleus, disrupts the role of REM sleep and also, presumably, the role of nonREM to REM sleep transitions in the consolidation of contextual fear memory. Thus, both sleep spindles and P-waves mediate the effects of sleep on the consolidation of emotional memory.

Whether and how learning and emotional stimuli affect the quantity and quality of subsequent sleep may influence whether individuals develop PTSD. Spoormaker et al. (10.1007/s00221-014-3831-2) reveal that activity in the left ventromedial prefrontal cortex (vmPFC) during fear conditioning correlates with the subsequent rise in the amount of REM sleep. They further show that both vmPFC and the amount of REM sleep have a negative relationship with skin conductance fear expression to a conditioned stimulus. Their data show that REM sleep mediates the relationship between vmPFC activity during fear conditioning and impairments in fear extinction. Wellman et al. (10.1007/s00221-014-3850-z) blocked fear-induced reductions in REM sleep and consolidation of the fear memory itself by inactivating the basolateral amygdala prior to fear conditioning. Thus, the prefrontal cortex and amygdala both mediate the effects of fear on sleep and the effects of sleep on fear extinction recall.

The effects of sleep alterations on subsequent waking function are also revealed in this issue. Tkachenko et al. (10.1007/s00221-014-3827-y) show that psychopathologies such as anxiety and depression are correlated with self-reported sleep quality in the home, even in healthy volunteers. Individuals complaining of sleep problems showed higher scores on scales measuring somatic complaints, anxiety, and depression. A long-lasting negative association between sleep and psychopathological characteristics is thus demonstrated even within normal subjects.

The last paper by Vanderheyden, Liberzon, and Poe (10.1007/s00221-014-3890-4) presents a unifying view of non-REM sleep features and REM sleep features for the formation of emotional memories and the assignment of their contextual relevance. They propose that sleep uniquely serves to enhance the recognition of novel elements and provides the registration/updating process for these memories in the face of novel information. They further discuss the relevance of these processes to PTSD.

Indeed, several models have emerged trying to explain the interaction between sleep and waking behavior, some of them pointing to the participation of specific sleep stages, sleep features, and brain systems. What is ultimately revealed in this issue is the fundamental importance of sleep, REM sleep in particular, but also sleep spindles during non-REM sleep, in the processing of emotional memories and down-regulation of emotional reactivity. Proper recognition of contextual differences and higher cognitive processing contribute to the decision of whether to permit autonomic fear reactions or prevent extinguished stimuli, thereby attenuating emotional reactivity. Results of sleep impairments counter-indicate sleep disruption prior to and after threat exposure as it could increase susceptibility to PTSD. Further, design of interventions to reverse PTSD and other emotional disorders need take into account physiological features of sleep such as spindle density, theta amplitude, and gamma coherence.

The guest editors solicited manuscripts that were then peer-reviewed in the usual fashion. The guest editors are thankful to the authors for their contributions and to the reviewers for their helpful comments. We are also grateful to the editorial and production team of Experimental Brain Research for supporting this special issue. 